\title{
ENDOCRINE DISORDERS INDUCED BY ANTIEPILEPTIC DRUGS
}

Endocrine disturbances in adolescent women with epilepsy, and the effects of antiepileptic drugs (AEDs) and hormonal contraception are discussed by a pediatric neurologist and epileptologist at the Children's Hospital of Wisconsin, Milwaukee, WI. AEDs that induce hepatic microsomal enzymes and may interact adversely with hormonal contraception include carbamazepine, phenobarbital, phenytoin, and primidone. AEDs that do not interact adversely with hormonal contraception are the P-450 enzyme inhibitors, valproate and zonisamide, and non-inhibitors, gabapentin, lamotrigine, levetiracetam, and vigabatrin. Concurrent use of hormonal contraception and AEDs does not lead to increased seizure frequency. Increased estrogen doses $(>50 \mathrm{mcg})$ in patients treated with enzymeinducing AEDs may further reduce the risk of unintended pregnancy. AEDs that have no effect on hepatic microsomal enzymes are preferred. Treatment with enzyme-inducing AEDs may increase the risk of sexual dysfunction, irregular menstruation, and ovulatory failure in women with epilepsy. Treatment with the enzyme inhibitor valproate may increase the risk of anovulatory menstrual cycles, polycystic ovarian syndrome, and hyperinsulinemia. Enzymeinducing AEDs increase metabolism and protein binding of estrogen and progesterone, respectively, but do not significantly increase the failure rates of hormonal contraception. Counseling for adolescents on reproductive health issues is particularly important in patients with epilepsy. Supplements of folic acid, calcium, and vitamin D are recommended. (Zupanc ML. Antiepileptic drugs and hormonal contraceptives in adolescent women with epilepsy. Neurology March 2006;66(Suppl 3);S37-S45). (Reprints: Dr Mary L Zupanc, Children's Hospital of Wisconsin, 900 West Wisconsin Ave, PO Box 1997, Milwaukee, WI 53201).

COMMENT. While seizure control is the primary consideration, birth control and the inter-relation of AEDs and hormonal contraception are important in the treatment of the adolescent female patient with epilepsy. The frequency of depression in women with epilepsy and the need for early identification of at risk patients is emphasized in this supplement concerning the adolescent female with epilepsy. (Harden CL et al. Neurology 2006;66(Suppl 3);S3-S4).

\section{ATTENTION DEFICIT DISORDERS}

\section{PERVASIVE DEVELOPMENTAL DISORDER AND ADHD}

The relationship between patients with attention deficit hyperactivity disorder (ADHD) and those with pervasive developmental disorders (PDD) was studied at Okayama University Graduate School of Medicine, Japan. Using the High-Functioning Autism Spectrum Screening Questionnaire (ASSQ) and ADHD Rating Scale-IV, the ASSQ scores of the PDD group ( 9 boys and 6 girls; 5-13 years) and ADHD group (19 boys and 1 girl; 5-15 years) were significantly higher than the control normal group (38 boys and 2 girls; 5-15 years); and the PDD group scored higher than the ADHD group. The WISC-III Full Scale IQ was average and similar in the 2 test groups. Both groups had higher scores than controls in each of the three behavioral disorders on the ASSQ: reciprocal social interaction, verbal and 